\title{
The Selective Estrogen Receptor Modulator Raloxifene Inhibits Neutrophil Extracellular Trap Formation
}

\author{
Roxana Flores ${ }^{1}$, Simon Döhrmann ${ }^{1}$, Christina Schaal ${ }^{2}$, Abdul Hakkim $^{3}$, Victor Nizet ${ }^{1}$ and \\ Ross Corriden ${ }^{4 *}$ \\ 'Department of Pediatrics, Division of Host-Microbe Systems and Therapeutics, University of California San Diego, La Jolla, \\ CA, USA, ${ }^{2}$ Department of Physiological Chemistry, University for Veterinary Medicine Hannover, Hanover, Germany, \\ ${ }^{3}$ Department of Molecular Biology, Massachusetts General Hospital, Harvard Medical School, Boston, MA, USA, \\ ${ }^{4}$ Department of Pharmacology, University of California San Diego, La Jolla, CA, USA
}

OPEN ACCESS

Edited by:

Mariana Julieta Kaplan, National Institute of Arthritis and Musculoskeletal and Skin Diseases, USA

Reviewed by: Winfried Barchet, University of Bonn, Germany Philippe Georgel, University of Strasbourg, France

*Correspondence: Ross Corriden rcorriden@ucsd.edu

Specialty section: This article was submitted to Molecular Innate Immunity, a section of the journal Frontiers in Immunology

Received: 13 August 2016 Accepted: 22 November 2016 Published: 07 December 2016

Citation:

Flores R, Döhrmann S, Schaal C, Hakkim A, Nizet $V$ and Corriden $R$ (2016) The Selective Estrogen

Receptor Modulator Raloxifene Inhibits Neutrophil Extracellular Trap

Formation.

Front. Immunol. 7:566. doi: 10.3389/fimmu.2016.00566
Raloxifene is a selective estrogen receptor modulator typically prescribed for the prevention/treatment of osteoporosis in postmenopausal women. Although raloxifene is known to have anti-inflammatory properties, its effects on human neutrophils, the primary phagocytic leukocytes of the immune system, remain poorly understood. Here, through a screen of pharmacologically active small molecules, we find that raloxifene prevents neutrophil cell death in response to the classical activator phorbol 12-myristate 13-acetate (PMA), a compound known to induce formation of DNA-based neutrophil extracellular traps (NETs). Inhibition of PMA-induced NET production by raloxifene was confirmed using quantitative and imaging-based assays. Human neutrophils from both male and female donors express the nuclear estrogen receptors ER $\alpha$ and $E R \beta$, known targets of raloxifene. Similar to raloxifene, selective antagonists of these receptors inhibit PMA-induced NET production. Furthermore, raloxifene inhibited PMA-induced ERK phosphorylation, but not reactive oxygen species production, pathways known to be key modulators of NET production. Finally, we found that raloxifene inhibited PMAinduced, NET-based killing of the leading human bacterial pathogen, methicillin-resistant Staphylococcus aureus. Our results reveal that raloxifene is a potent modulator of neutrophil function and NET production.

Keywords: neutrophils, raloxifene, MRSA, host-pathogen interactions, extracellular traps

\section{INTRODUCTION}

Neutrophil extracellular traps (NETs), first described in 2004 (1), are DNA-based structures decorated with antibacterial components (e.g., histones, antimicrobial peptides, and myeloperoxidase) that form during a specialized cell death pathway (NETosis) to ensnare and kill pathogens. Since their initial discovery, NETs have been shown to play an important role in host defense; however, the molecular mechanisms that control NET production remain a topic of active investigation. Although NET production is typically driven in concert with the generation of reactive oxygen species (ROS) and activation of PKC, additional pathways that facilitate NET production (e.g., ERK activation, histone citrullination, and intracellular ceramide accumulation) have been identified in recent years (2-4). Identification of novel pathways that modulate NETosis could reveal new therapeutic targets to enhance antibacterial innate immunity or inhibit aberrant neutrophilmediated inflammatory responses. 
Recently, we demonstrated that tamoxifen, an estrogen receptor modulator classically described as an estrogen receptor antagonist, is a potent NET inducer (4). Our results indicated that tamoxifen-induced NET production is largely driven by an accumulation of intracellular ceramide resulting from inhibition of glucosylceramide synthase. This finding prompted us to investigate whether other selective estrogen receptor modulators (SERMs) with pharmacological properties distinct from tamoxifen may also modulate neutrophil function/NET production. Of immediate interest was raloxifene, an FDA-approved compound typically prescribed for the prevention or treatment of osteoporosis in postmenopausal women (5). Similar to tamoxifen, the most well-characterized pharmacological targets of raloxifene are the nuclear estrogen receptors $\operatorname{ER} \alpha$ and $\operatorname{ER} \beta$ (6). Although raloxifene has been described to exhibit potent anti-inflammatory effects (7), our understanding of how it affects neutrophil function remains limited (8), with no studies having directly assessed its effects on human neutrophils. Intriguingly, however, raloxifene treatment reduces plasma neutrophil myeloperoxidase levels in vivo (9). Because myeloperoxidase is released into the plasma following NET induction (10), this association suggested that raloxifene may inhibit NET production. Here, we provide evidence that raloxifene prevents neutrophil cell death in response to treatment with the NET inducer phorbol 12-myristate 13-acetate (PMA). Building on these results, we use both quantitative and imaging-based approaches to define the effects of raloxifene on NET production and probe the molecular mechanisms underlying this effect.

\section{MATERIALS AND METHODS}

\section{Materials}

Raloxifene hydrochloride, MPP dihydrochloride, and PHTTP were purchased from Tocris Bioscience (Bristol, UK). PMA, micrococcal nuclease from Staphylococcus aureus, the small molecule library used for screening and all other compounds were obtained from Sigma-Aldrich (St. Louis, MO, USA).

\section{Cell Viability Screen}

An image-based screen, described in detail in Ref. (2), was used in conjunction with the Sigma LOPAC (Library of Pharmacologically Active Compounds; Sigma-Aldrich, St. Louis, MO, USA) library of $~ 1280$ pharmacologically active small molecules. Following exposure to compounds from this library (each at a final concentration of $100 \mu \mathrm{M}$ ), cell viability in response to 4-h PMA treatment was quantified by fixing cells with $2 \%$ paraformaldehyde, staining nuclei with the fluorescent DNA stain Sytox Green $(2 \mu \mathrm{M}$; Life Technologies, Carlsbad, CA, USA), and imaging using an Ascent Fluoroskan MTP reader (Thermo Scientific).

\section{Neutrophil Isolation}

Venous blood was collected from healthy volunteers according to an approved protocol. Heparin was used as an anticoagulant. Neutrophils were isolated using Polymorphprep density gradient medium (Axis-Shield, Dundee, Scotland) according to the manufacturer's protocol.

\section{Fluorescence Microscopy/ NET Visualization}

To visualize NETs, cells were seeded in Nunc Lab-Tek II Chambered Coverglass slides (Thermo Fisher, Waltham, MA, USA) at a density of $2 \times 10^{5}$ cells/well. Following incubation with indicated antagonist compounds, NET production was induced via addition of $25 \mathrm{nM}$ PMA prior to a 2 -h incubation at $37^{\circ} \mathrm{C}$ with $5 \% \mathrm{CO}_{2}$. Cells were fixed by addition of paraformaldehyde (4\% final) for $10 \mathrm{~min}$ at $24^{\circ} \mathrm{C}$. Following three washes with PBS, neutrophils were permeabilized via incubation in Triton X-100 solution $(0.1 \%)$ for $10 \mathrm{~min}$ at $24^{\circ} \mathrm{C}$. After an additional three washes with PBS, DNA was stained with $2 \mu \mathrm{M}$ Sytox Green (Life Technologies, Carlsbad, CA, USA) for $10 \mathrm{~min}$ at $24^{\circ} \mathrm{C}$; slides were then washed a final three times with $\mathrm{PBS}$ prior to imaging using a Zeiss AxioObserver D1 microscope equipped with an LD A-Plan 20X/0.35 Ph1 objective (Carl Zeiss AG, Oberkochen, Germany).

Imaging of intracellular ceramide was performed by permeabilizing cells with $0.25 \%$ Triton X-100 prior to fixation with paraformaldehyde, blocking with PBS containing $2 \%$ bovine serum albumin (2\% PBS-BSA), and 2\% donkey serum for $1 \mathrm{~h}$. Cells were subsequently incubated for $1 \mathrm{~h}$ with mouse anti-ceramide primary antibody (1:300 in 2\% PBS-BSA; Sigma-Aldrich, St. Louis, MO, USA) and $45 \mathrm{~min}$ (protected from light) with Alexa Fluor 488 donkey anti-mouse IgG secondary antibody (1:500 in $2 \%$ PBS-BSA; Life Technologies, Carlsbad, CA, USA). Representative images shown were collected the Zeiss AxioObserver D1 microscope and objective described above, with exposure and gain settings kept consistent during collection of control and raloxifene-treated images.

\section{PicoGreen NET Quantification Assay}

All incubations were performed at $37^{\circ} \mathrm{C}$ and $5 \% \mathrm{CO}_{2}$ unless otherwise noted. Isolated neutrophils were plated on 96 -well tissue culture plates at $2 \times 10^{5}$ cells/well. Cells were pretreated with estrogen receptor antagonists (e.g., raloxifene) for $30 \mathrm{~min}$, then incubated an additional $2 \mathrm{~h}$ with PMA $(25 \mathrm{nM})$ to induce NET production. Micrococcal nuclease was then added at a final concentration of $500 \mathrm{mU} / \mathrm{ml}$ for $10 \mathrm{~min}$ to allow digestion of extracellular DNA. Following addition of $5 \mathrm{mM}$ EDTA, plates were centrifuged at $200 \times g$ for $8 \mathrm{~min}$; supernatant samples $(100 \mu \mathrm{l})$ were then collected and transferred to a 96-well plate. DNA was quantified using a Quant-iT PicoGreen ${ }^{\circledR}$ dsDNA Assay Kit from Life Technologies (Carlsbad, CA, USA).

\section{Quantification of Estrogen Receptor Expression}

Freshly isolated neutrophils were harvested and $1 \times 10^{6}$ cells resuspended in TRIzol Reagent (Thermo Fisher Scientific, Waltham, MA, USA). Total RNA was extracted using a Zymo Research Direct-zol RNA kit (Irvine, CA, USA) following the manufacturer's protocol (including DNAse treatment). Following quantification of RNA using a Thermo Fisher Scientific Nanodrop Spectrophotometer (Waltham, MA, USA), quantitative PCR was performed on an ABI 7000 platform using USB $^{\circledR}$ Veriquest $^{\mathrm{TM}}$ Probe One-Step qRT-PCR Master Mix $(2 \times)$. Predesigned human TaqMan ${ }^{\circledR}$ Gene Expression Assays (Life Technologies, Carlsbad, 
CA, USA) were used to probe for expression of ESRRG, ESR2, and GAPDH. An equal input of total RNA was used for each assay.

\section{Transwell Chemotaxis Assay}

Neutrophils, pre-incubated for $20 \mathrm{~min}$ at $37^{\circ} \mathrm{C}$ in $\mathrm{HBSS}$ alone or HBSS with $10 \mu \mathrm{M}$ raloxifene, were seeded in 6-mm transwell permeable supports ( $3-\mu \mathrm{m}$ pore size; Corning Inc., Corning, NY, USA) that were placed in 24-well plates; lower chambers contained either HBSS alone or $100 \mathrm{nM}$ f-Met-Leu-Phe (fMLP). Following a 45 -min incubation at $37^{\circ} \mathrm{C}$, inserts were removed, and cells were lysed by addition of Triton X-100 (0.1\% final, $\left.10 \mathrm{~min}, 24^{\circ} \mathrm{C}\right)$. To determine the relative levels of migration to the lower well, the colorimetric elastase substrate $N$-methoxysuccinyl-Ala-Ala-ProVal $p$-nitroanilide was added to lysed cell samples (10 $\mathrm{mM}$ final); after a 30 -min incubation at $24^{\circ} \mathrm{C}$, absorbance at $405 \mathrm{~nm}$ was measured using a SpectraMAX Gemini EM fluorescence reader (Molecular Devices, Sunnyvale, CA, USA).

\section{Phagocytosis Assay}

Following a 30 -min incubation at $37^{\circ} \mathrm{C}$ in the presence or absence of $10 \mu \mathrm{M}$ raloxifene, neutrophils were combined in a 96-well plate $\left(2 \times 10^{5}\right.$ cells/well $)$ with pHrodo Red $S$. aureus Bioparticles (Life Technologies, Carlsbad, CA, USA) as specified by the manufacturer. Plates were incubated at $37^{\circ} \mathrm{C}$, and phagocytosis was assessed by measuring fluorescence intensity ( $560 \mathrm{~nm}$ excitation, $585 \mathrm{~nm}$ emission) at $15 \mathrm{~min}$ intervals using a SpectraMAX Gemini EM fluorescence reader (Molecular Devices, Sunnyvale, CA, USA).

\section{ROS Production Assays}

Neutrophils were incubated in HBSS supplemented with $10 \mathrm{mM}$ $2^{\prime}, 7^{\prime}$-dichlorofluorescein diacetate (DCFDA) for $20 \mathrm{~min}$ at $37^{\circ} \mathrm{C}$ with gentle agitation. Neutrophils were then centrifuged at $400 \times g$ for $5 \mathrm{~min}$, washed with HBSS, and centrifuged again using the same settings before being counted and resuspended in HBSS at a concentration of $5 \times 10^{6}$ cells $/ \mathrm{ml}$. Samples $(100 \mu \mathrm{l})$ of cell suspension were then added to a 96 -well plate $\left(5 \times 10^{5}\right.$ cells/ well) and incubated with HBSS or raloxifene for 30 min prior to addition of PMA. Fluorescence intensity (485 nm excitation, $530 \mathrm{~nm}$ emission) was measured at 15 -min intervals using a SpectraMAX Gemini EM fluorescence reader over $2 \mathrm{~h}$; between reads, plates were incubated at $37^{\circ} \mathrm{C}$ while protected from light.

\section{Total/Phospho-ERK ELISA}

Relative levels of total/phospho-ERK were determined using InstantOne ELISA kits (eBiosciences, San Diego, CA, USA) according to the manufacturer's protocol, with minor modifications (11). Total/phospho ERK was quantified following PMA stimulation $(25 \mathrm{nM})$ for $45 \mathrm{~min}$ in the presence or absence of raloxifene $(10 \mu \mathrm{M})$.

\section{NET Killing Assay}

Neutrophils suspended in serum-free RPMI (SF-RPMI) were added to 48 -well plates at a density of $4 \times 10^{5}$ cells/well. SF-RPMI or raloxifene $(10 \mu \mathrm{M}$ final) was added to applicable wells, and cells were incubated for $30 \mathrm{~min}$ at $37^{\circ} \mathrm{C}$ with $5 \% \mathrm{CO}_{2}$ (identical wells containing no neutrophils were also prepared). Following addition of PMA to applicable wells, cells were incubated for a further $4 \mathrm{~h}$ at $37^{\circ} \mathrm{C}$ with $5 \% \mathrm{CO}_{2}$. Overnight cultures of USA300 MRSA (strain UAMS 1182) bacteria were resuspended in RPMI containing $10 \% 70^{\circ} \mathrm{C}$ heat-inactivated fetal bovine serum (FBS) to achieve a density of $8 \times 10^{5}$ colony forming units (CFUs) $/ \mathrm{ml}$. Fifty microliters of bacterial suspension were added to each well, resulting in a $2 \%$ final concentration of FBS and a multiplicity of infection (MOI) of 0.1 . Following a centrifugation at $1600 \mathrm{rpm}$ for $5 \mathrm{~min}$, mixed neutrophil/bacterial cultures were incubated for $15 \mathrm{~min}$ at $37^{\circ} \mathrm{C}$ with $5 \% \mathrm{CO}_{2}$. Samples from each well were then collected, serially diluted in sterile $\mathrm{H}_{2} \mathrm{O}$, and plated on Todd Hewitt Agar for enumeration of CFUs.

\section{Statistical Analysis}

All statistical analyses described in the figure legends were performed using GraphPad Prism version 7.0.

\section{RESULTS}

\section{Raloxifene Inhibits PMA-Induced NET Production}

An image-based cell viability screen (2) using the fluorescent DNA dye Sytox Green identified raloxifene as one of the small molecules from the $\sim 1200$ pharmacologically active known compounds tested to have enhanced the survival of neutrophils when exposed to PMA, a potent NET inducer (Figure 1A), suggesting that raloxifene treatment may inhibit NET production.
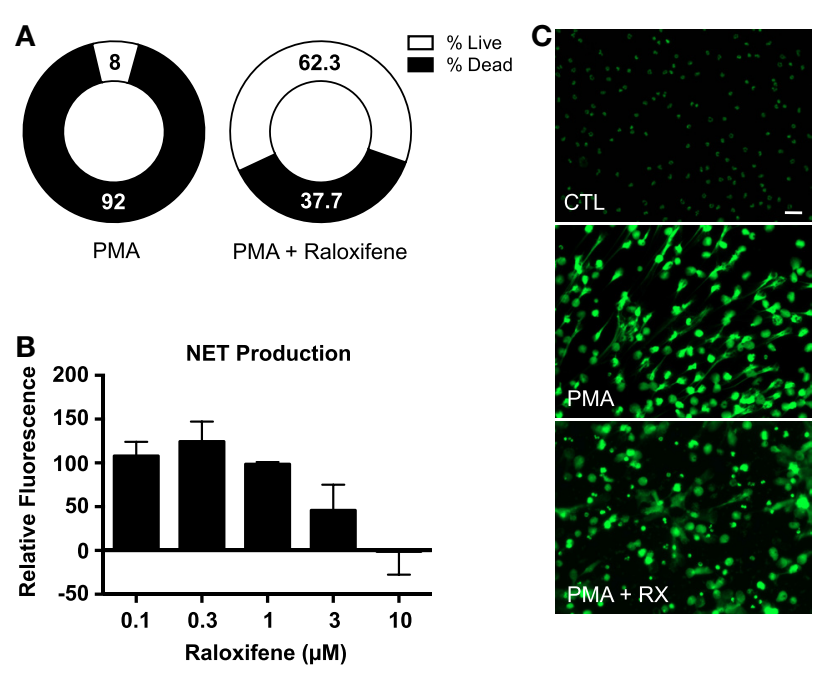

FIGURE 1 | Raloxifene inhibits PMA-induced NET production. (A) A plate reader-based live-dead screening assay was used to assess the affects of raloxifene on PMA-induced cell death. (B) PMA-induced NET production was quantified in cells pre-incubated with raloxifene at the indicated concentrations; PicoGreen DNA dye was used to measure extracellular DNA (NET production expressed as a percent of PMA-only control; $n=5$ ). (C) Visualization of NET production by control (CTL) or PMA-induced NETs in the presence or absence of raloxifene $(3 \mu \mathrm{M})$. DNA/NETs stained with Sytox Green. Data shown are expressed as mean values \pm SE of the indicated number of biological replicates (each performed in triplicate). Where applicable, results were analyzed by one-way ANOVA. 
To directly assess the effect of raloxifene on NET production, we quantified release of DNA using the fluorescent DNA dye PicoGreen, finding a concentration-dependent inhibition of PMA-induced NET production by the drug (Figure 1B). These results were confirmed via fluorescence microscopy of SYTOX Green-stained cells, which revealed a reduction in the number of NET-positive cells and an increase in the number of intact, condensed nuclei (Figure 1C).

\section{Raloxifene Does Not Affect Neutrophil Chemotaxis or Phagocytosis but Reduces Intracellular Ceramide}

We previously demonstrated that tamoxifen, in addition to stimulating NET production, enhances both chemotaxis in a chemoattractant gradient and phagocytosis of bacteria-labeled bioparticles (4). Tamoxifen treatment also increases intracellular ceramide levels in neutrophils via inhibition of glucosylceramide synthase, a key enzyme in the sphingolipid synthesis pathway that converts ceramide to glucosylceramide. Tamoxifen-induced NET production is dependent on this increase of intracellular ceramide (4). In contrast to our observations with tamoxifen, we found that raloxifene had no statistically significant effect on either chemotaxis in a gradient of the chemoattractant fMLP (Figure 2A) or phagocytosis of $S$. aureus-labeled bioparticles (Figure 2B). Furthermore, immunostaining of raloxifene-treated neutrophils revealed that, in contrast to tamoxifen, raloxifene reduces intracellular ceramide levels (Figures 2C,D).

\section{Selective Estrogen Receptor Antagonists Inhibit PMA-Induced NET Production}

Quantitative PCR-based analysis of ER $\alpha$ and $\operatorname{ER} \beta$ expression in neutrophils from 12 healthy donors (6 male and 6 female) revealed that circulating neutrophils express both nuclear receptors (Figure 3A). Fluorescence-based quantification of NET production indicated that the selective estrogen receptor antagonists MPP (ER $\alpha$ inhibitor) and PHTPP (ER $\beta$ inhibitor) mimicked the raloxifene-mediated inhibition of PMA-induced NET production (Figure 3B). Imaging of SYTOX Green-stained cells revealed some residual NET production by neutrophils pre-incubated with MPP and PHTPP, suggesting the potential involvement of multiple estrogen receptors in raloxifene-mediated inhibition of NETosis (Figure 3C).

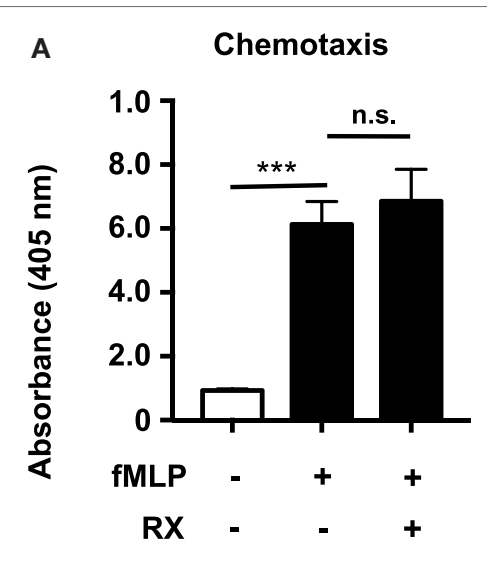

C

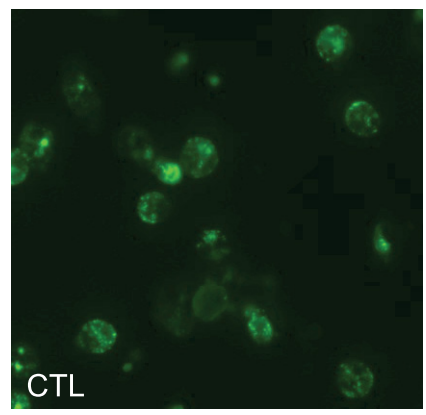

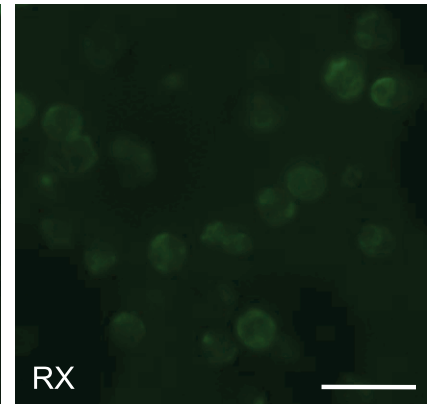

B
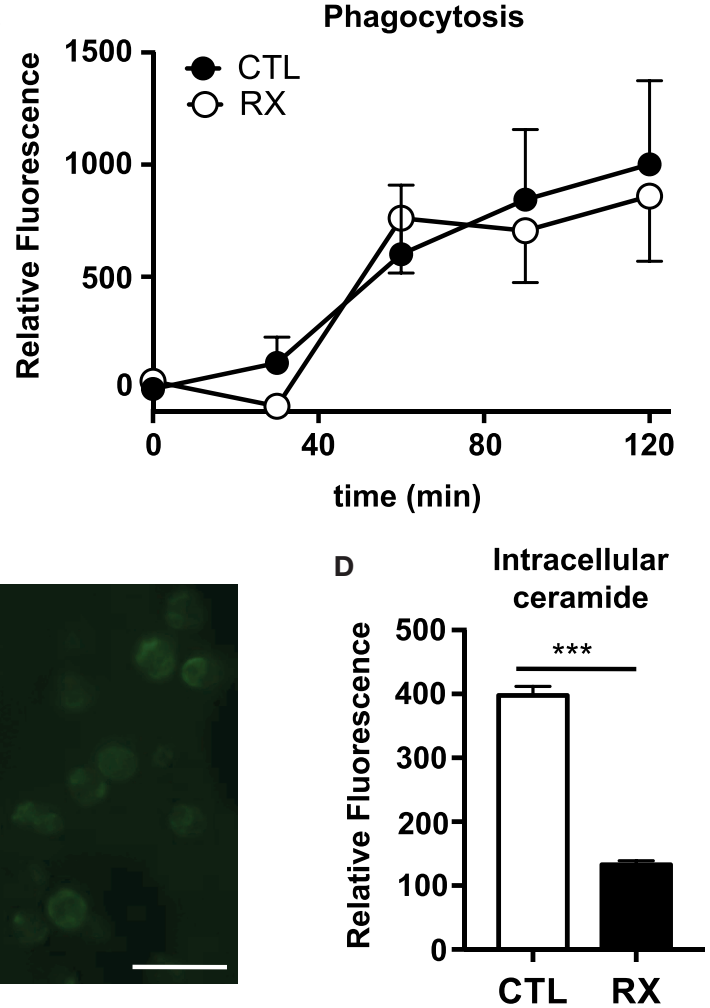

FIGURE 2 | Raloxifene does not affect neutrophil chemotaxis or phagocytosis but reduces intracellular ceramide. (A) Transwell chemotaxis assays were used to quantify neutrophil migration in a gradient of the chemottractant fMLP (100 nM) in the presence or absence of $10 \mu \mathrm{M}$ raloxifene ( $n=3)$. (B) Neutrophils were incubated with $\mathrm{pH}$-sensitive $\mathrm{S}$. aureus-labeled bioparticles in the presence or absence of $10 \mu \mathrm{M}$ raloxifene $(n=3)$. (C) An immunostaining approach was used to visualize ceramide accumulation in untreated control (CTL) or raloxifene-treated (RX; 10) neutrophils (45 min incubation); representative images from three separate experiments are shown. (D) Mean fluorescence intensity (MFI) values were collected using region of interest analysis to quantify relative ceramide levels in control and $10 \mu \mathrm{M}$ raloxifene-treated neutrophils. Results shown represent average MFI values for 96-193 cells imaged using identical settings in three separate experiments. Where applicable, results were analyzed by one-way ANOVA and post-hoc Dunnett's test or Student's $t$-test. ${ }^{\star \star \star} P<0.001$ vs. control values. 


\section{Raloxifene Inhibits PMA-Induced ERK Phosphorylation but Not PMA-Induced ROS Production}

Reactive oxygen species production was quantified using the membrane-permeable fluorescent ROS probe $2^{\prime}, 7^{\prime}$-dichlorofluorescein diacetate (DCFDA). Although PMA-induced NET production is known to be ROS dependent (1), raloxifene did not inhibit PMA-induced ROS production (Figures 4A,B). However, ELISA-based quantification of ERK phosphorylation revealed that raloxifene significantly inhibited PMA-induced
ERK activation (Figure 4C), a critical signaling event that has been shown to play a role in NET production (2).

\section{Raloxifene Inhibits NET-Mediated Killing of Methicillin-Resistant Staphylococcus aureus}

To determine whether raloxifene affects NET-based bactericidal activity of neutrophils, we performed a NET-based killing assay in which neutrophils were pre-stimulated with PMA to induce NET production prior to challenge with methicillin-resistant
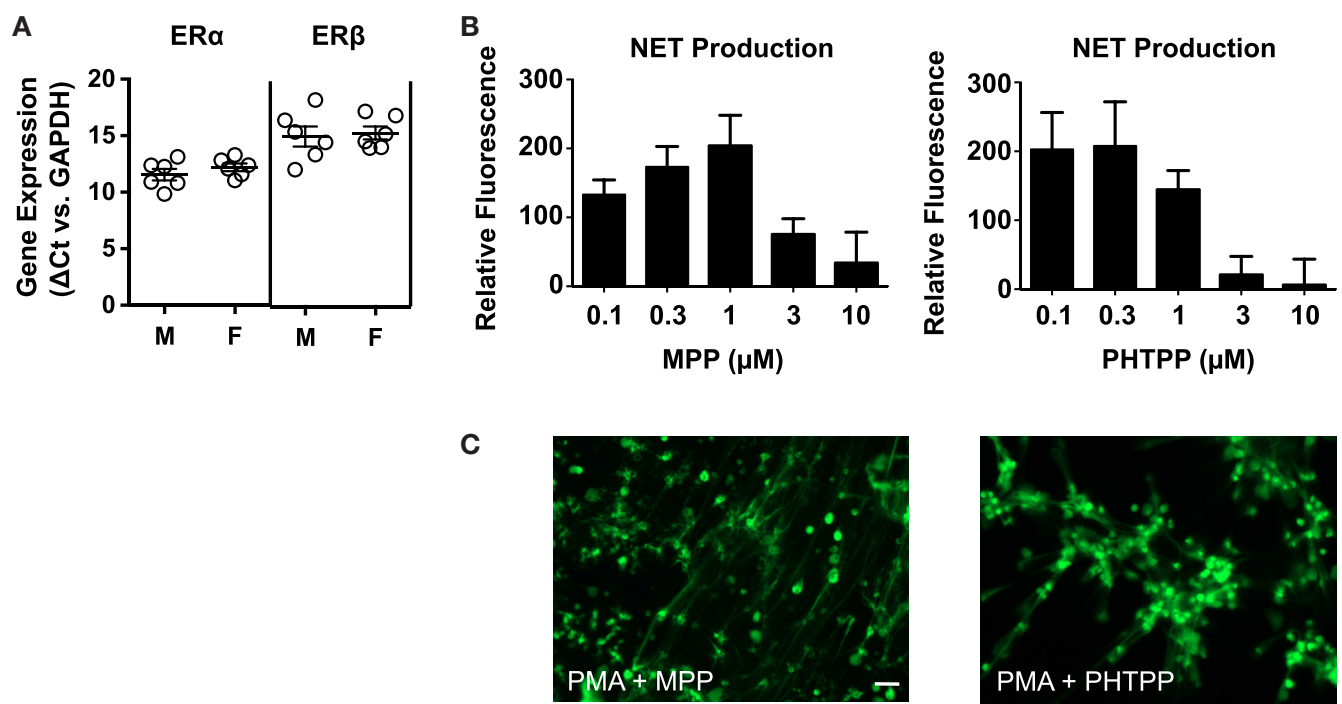

FIGURE 3 | Selective antagonists of ER $\alpha$ and ER $\beta$ inhibit PMA-induced NET production. (A) Quantitative PCR was used to measure ER $\alpha$ and ER $\beta$ gene expression in neutrophils from 12 healthy donors [6 males (M), 6 females (F)]. Data expressed as $\Delta$ Ct values relative to GAPDH. (B) PMA-induced NET production was quantified in cells pre-incubated with MPP (selective ER $\alpha$ antagonist) or PHTPP (selective ER $\beta$ antagonist) at the indicated concentrations; PicoGreen DNA dye was used to measure extracellular DNA (NET production expressed as a percent of PMA-only control; $n=5$ ). (C) Visualization of PMA-induced NET production in cells pre-incubated with MPP or PHTPP. DNA/NETs stained with Sytox Green. Data shown are expressed as mean values \pm SE of the indicated number of biological replicates (each performed in triplicate). Where applicable, results were analyzed by one-way ANOVA and post hoc Newman-Keuls test.
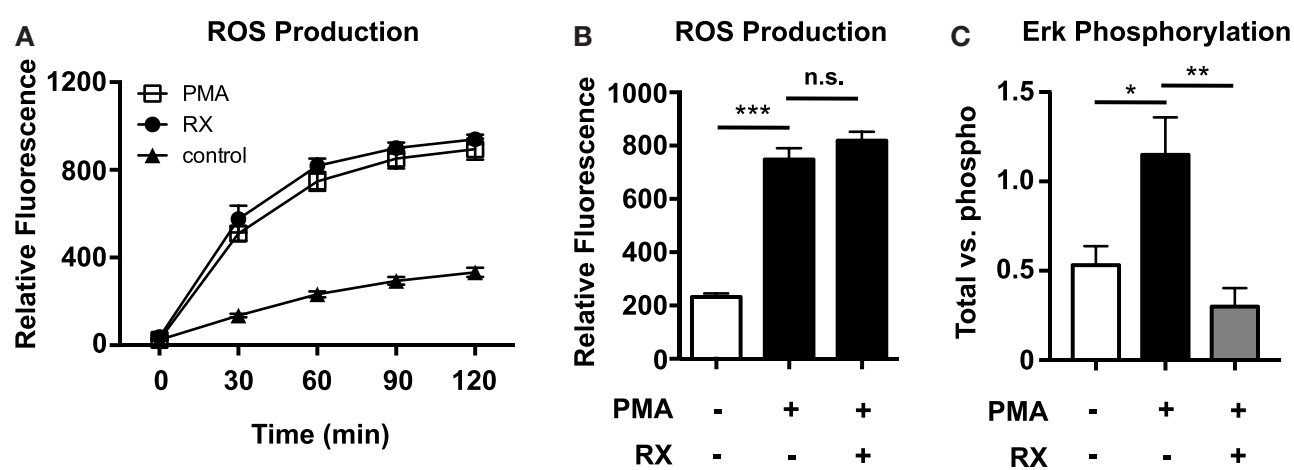

FIGURE 4 | Raloxifene inhibits PMA-induced ERK phosphorylation, but not PMA-induced ROS production. ROS production in control and 25 nM PMA-treated neutrophils in the presence/absence of raloxifene $(10 \mu \mathrm{M})$ was quantified over time (A) and at $2 \mathrm{~h}$ (B) using the cell-permeable fluorescent ROS probe DCFDA $(n=3)$. (C) PMA-induced ERK phosphorylation in the presence/absence of $10 \mu \mathrm{M}$ raloxifene was quantified using total/phospho ELISAs ( $n=4)$. Data shown are expressed as mean values \pm SE of the indicated number of biological replicates (each performed in duplicate or triplicate). Where applicable, results were analyzed by one-way ANOVA and post hoc Newman-Keuls test. ${ }^{\star} P<0.05$, ${ }^{\star \star \star} P<0.001$ vs. control values. 
Staphylococcus aureus (MRSA; MOI 0.1). Stimulation of NETs via $4 \mathrm{~h}$ incubation with $25 \mathrm{nM}$ PMA produced a statistically significant reduction in bacterial survival in neutrophil/MRSA cocultures (Figure 5). Incubation with raloxifene $30 \mathrm{~min}$ prior to addition of PMA reversed this effect.

\section{DISCUSSION/CONCLUSION}

Here, we show evidence that the SERM raloxifene inhibits PMA-induced NET production. Unlike tamoxifen, an estrogen receptor modulator that induces NET production (4), raloxifene reduces intracellular ceramide levels. Neutrophils from both male and female donors express the nuclear estrogen receptors ER $\alpha$ and $\operatorname{ER} \beta$, the best characterized targets of raloxifene. Selective antagonists of either receptor inhibited PMA-induced NETosis, suggesting that raloxifene may act through multiple receptors to inhibit NET production, and further indicating that tamoxifeninduced NET production is largely dependent on estrogen

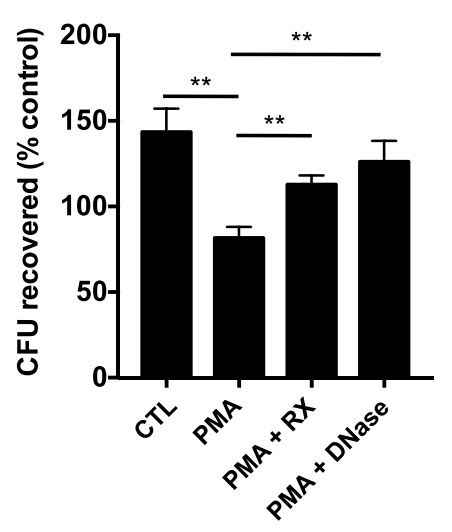

FIGURE 5 | Raloxifene inhibits NET-mediated killing of methicillinresistant Staphylococcus aureus. Neutrophil killing of USA300 MRSA was quantified in a NET-based killing assay. Control (CTL) represents bacteria exposed to unstimulated neutrophils and PMA represents neutrophils treated with $25 \mathrm{nM}$ PMA. Raloxifene $(\mathrm{RX} ; 10 \mu \mathrm{M})$ or DNase $(7.5 \mathrm{U} / \mathrm{ml})$ were added 30 min prior or $3.5 \mathrm{~h}$ following addition of PMA, respectively $(n=3)$. Data shown are expressed as mean values \pm SE of three independent experiments, each performed in triplicate. Results were analyzed by Student's $t$-test. ${ }^{\star \star} P<0.01$ vs. control values.

\section{REFERENCES}

1. Brinkmann V, Reichard U, Goosmann C, Fauler B, Uhlemann Y, Weiss DS, et al. Neutrophil extracellular traps kill bacteria. Science (2004) 303(5663):1532-5. doi:10.1126/science. 1092385

2. Hakkim A, Fuchs TA, Martinez NE, Hess S, Prinz H, Zychlinsky A, et al. Activation of the Raf-MEK-ERK pathway is required for neutrophil extracellular trap formation. Nat Chem Biol (2011) 7(2):75-7. doi:10.1038/ nchembio. 496

3. Li P, Li M, Lindberg MR, Kennett MJ, Xiong N, Wang Y. PAD4 is essential for antibacterial innate immunity mediated by neutrophil extracellular traps. J Exp Med (2010) 207(9):1853-62. doi:10.1084/jem.20100239

4. Corriden R, Hollands A, Olson J, Derieux J, Lopez J, Chang JT, et al. Tamoxifen augments the innate immune function of neutrophils through modulation of intracellular ceramide. Nat Commun (2015) 6:8369. doi:10.1038/ ncomms 9369

receptor-independent, non-specific effects (e.g., inhibition of glucosylceramide synthase). We find that raloxifene inhibits PMA-induced ERK phosphorylation, a signal transduction event known to be critical for NETosis (2); thus, raloxifene likely modulates NET production via regulation of ERK signaling.

Our results suggest that raloxifene may have beneficial therapeutic effects in contexts where excessive NETosis is undesirable and associated inflammatory changes cause damage to the host (12). NETs appear to play a role in the pathophysiology of rheumatoid arthritis (13); notably, raloxifene has been shown to exhibit significant antiarthritic properties in a murine model of this disease (14). The incidence of rheumatoid arthritis in raloxifene-treated postmenopausal women (and specifically the contribution of NETs to disease pathophysiology) represents an important area for further investigation; however, our results also suggest that raloxifene therapy may come at some consequence to patients at high risk of infection, as raloxifene inhibits NETmediated bacterial killing.

These findings provide new insight into the effects of raloxifene on neutrophil function, which at present are poorly understood. Such effects hold important clinical implications, given the large number of patients receiving this drug. Further exploration of the pathways mediating the anti-NETosis activity of raloxifene may reveal new therapeutic targets and facilitate development of therapies that can more selectively regulate NET production in appropriate physiological contexts.

\section{ETHICS STATEMENT}

All studies described here were reviewed and approved by the local (UCSD) Institutional Review Board.

\section{AUTHOR CONTRIBUTIONS}

RC and VN conceptualized and led the project. RC, RF, SD, CS, and $\mathrm{AH}$ designed and performed experiments and interpreted data. RC, RF, and VN wrote the manuscript.

\section{FUNDING}

This research was supported by NIH grants U54 HD071600, HD071600, AI057153, and AI052453 (to VN).

5. Hansdóttir H. Raloxifene for older women: a review of the literature. Clin Interv Aging (2008) 3(1):45-50. doi:10.2147/CIA.S224

6. Muchmore DB. Raloxifene: a selective estrogen receptor modulator (SERM) with multiple target system effects. Oncologist (2000) 5(5):388-92. doi:10.1634/ theoncologist.5-5-388

7. Sin-Ae L, Seok Hee P, Byung-Chul K. Raloxifene, a selective estrogen receptor modulator, inhibits lipopolysaccharide-induced nitric oxide production by inhibiting the phosphatidylinositol 3-kinase/Akt/nuclear factor-kappa B pathway in RAW264.7 macrophage cells. Mol Cells (2008) 26(1):48-52.

8. Zuckerman AH, Bryan N. Inhibition of LDL oxidation and myeloperoxidase dependent tyrosyl radical formation by the selective estrogen receptor modulator raloxifene (LY139481 HCL). Atherosclerosis (1996) 126(1):65-75. doi:10.1016/0021-9150(96)05894-7

9. Chung MT, Cheng PY, Lam KK, Chen SY, Ting YF, Yen MH, et al. Cardioprotective effects of long-term treatment with raloxifene, a selective estrogen receptor modulator, on myocardial ischemia/reperfusion injury 
in ovariectomized rats. Menopause (2010) 17(1):127-34. doi:10.1097/ gme.0b013e3181b4c4ac

10. Chowdhury CS, Giaglis S, Walker UA, Buser A, Hahn S, Hasler P. Enhanced neutrophil extracellular trap generation in rheumatoid arthritis: analysis of underlying signal transduction pathways and potential diagnostic utility. Arthritis Res Ther (2014) 16(3):R122. doi:10.1186/ar4579

11. Hollands A, Corriden R, Gysler G, Dahesh S, Olson J, Ali SR, et al. Natural product anacardic acid from cashew nut shells stimulates neutrophil extracellular trap production and bactericidal activity. J Biol Chem (2016) 291(27):13964-73. doi:10.1074/jbc.M115.695866

12. Kaplan MJ, Radic M. Neutrophil extracellular traps: double-edged swords of innate immunity. J Immunol (2012) 189(6):2689-95. doi:10.4049/ jimmunol.1201719

13. Khandpur R, Carmona-Rivera C, Vivekanandan-Giri A, Gizinski A, Yalavarthi S, Knight JS, et al. NETs are a source of citrullinated autoantigens and stimulate inflammatory responses in rheumatoid arthritis. Sci Transl Med (2013) 5(178):178ra40. doi:10.1126/scitranslmed.3005580
14. Jochems C, Islander U, Kallkopf A, Lagerquist M, Ohlsson C, Carlsten H. Role of raloxifene as a potent inhibitor of experimental postmenopausal polyarthritis and osteoporosis. Arthritis Rheum (2007) 56(10):3261-70. doi:10.1002/ art. 22873

Conflict of Interest Statement: The authors declare that the research was conducted in the absence of any commercial or financial relationships that could be construed as a potential conflict of interest.

Copyright (c) 2016 Flores, Döhrmann, Schaal, Hakkim, Nizet and Corriden. This is an open-access article distributed under the terms of the Creative Commons Attribution License (CC BY). The use, distribution or reproduction in other forums is permitted, provided the original author(s) or licensor are credited and that the original publication in this journal is cited, in accordance with accepted academic practice. No use, distribution or reproduction is permitted which does not comply with these terms. 